\title{
The prevalence, antibiotic resistance and $m e c A$ characterization of coagulase negative staphylococci recovered from non-healthcare settings in London, UK
}

Zhen $\mathrm{Xu}^{1,2}$, Haroun N. Shah ${ }^{3}$, Raju Misra ${ }^{4}$, Jiazhen Chen ${ }^{5}$, Wenhong Zhang ${ }^{5}$, Yuting Liu' ${ }^{6}$, Ronald R. Cutler ${ }^{2}$ and Hermine V. Mkrtchyan ${ }^{2,7^{*}}$

\begin{abstract}
Background: Coagulase negative staphylococci (CONS) are important reservoirs of antibiotic resistance genes and associated mobile genetic elements and are believed to contribute to the emergence of successful methicillin resistant Staphylococcus aureus (MRSA) clones. Although, these bacteria have been linked to various ecological niches, little is known about the dissemination and genetic diversity of antibiotic resistant CoNS in general public settings.

Methods: Four hundred seventy-nine samples were collected from different non-healthcare/general public settings in various locations $(n=355)$ and from the hands of volunteers $(n=124)$ in London UK between April 2013 and Nov 2014.

Results: Six hundred forty-three staphylococcal isolates belonging to 19 staphylococcal species were identified. Five hundred seventy-two (94\%) isolates were resistant to at least one antibiotic, and only 34 isolates were fully susceptible. Sixty-eight (11\%) mecA positive staphylococcal isolates were determined in this study. SCCmec types were fully determined for forty-six isolates. Thirteen staphylococci (19\%) carried SCCmec V, followed by 8 isolates carrying SCCmec type I (2\%), 5 SCCmec type IV (7\%), 4 SCCmec type II (6\%), 1 SCCmec type III (2\%), 1 SCCmec type VI (2\%), and 1 SCCmec type VIII (2\%). In addition, three isolates harboured a new SCCmec type 1A, which carried combination of class A mec complex and ccr type 1.

MLST typing revealed that all S. epidermidis strains possess new MLST types and were assigned the following new sequence types: ST599, ST600, ST600, ST600, ST601, ST602, ST602, ST603, ST604, ST605, ST606, ST607 and ST608.

Conclusions: The prevalence of antibiotic resistant staphylococci in general public settings demonstrates that antibiotics in the natural environments contribute to the selection of antibiotic resistant microorganisms. The finding of various SCCmec types in non-healthcare associated environments indicates the complexity of SCCmec. We also report on new MLST types that were assigned for all S. epidermidis isolates, which demonstrates the genetic variability of these isolates.
\end{abstract}

Keywords: CoNS, Antibiotic resistance, SCCmec, MLST

* Correspondence: h.mkrtchyan@uel.ac.uk

${ }^{2}$ School of Biological and Chemical Sciences, Queen Mary University of

London, London, UK

${ }^{7}$ School of Health, Sport and Biosciences, University of East London, E1 4NS,

London, UK

Full list of author information is available at the end of the article

(c) The Author(s). 2018 Open Access This article is distributed under the terms of the Creative Commons Attribution 4.0 International License (http://creativecommons.org/licenses/by/4.0/), which permits unrestricted use, distribution, and reproduction in any medium, provided you give appropriate credit to the original author(s) and the source, provide a link to the Creative Commons license, and indicate if changes were made. The Creative Commons Public Domain Dedication waiver (http://creativecommons.org/publicdomain/zero/1.0/) applies to the data made available in this article, unless otherwise stated. 


\section{Background}

Staphylococci are the most frequently isolated nosocomial pathogens, accounting for $30 \%$ of hospital associated infections [1]. Despite, that the high virulence of $S$. aureus has been evidenced in many studies [2], it is believed that coagulase-negative staphylococci (CoNS) act as an important reservoir of antimicrobial resistance genes and resistance-associated mobile genetic elements, which can transfer between staphylococcal species. Among other CoNS, S. epidermidis, S. hominis and S. haemolyticus are often reported to be resistant to multiple antibiotics $[3,4]$.

The mecA gene responsible for methicillin resistance was first determined in S. aureus, however, many other staphylococcal species were found to also harbour it [5]. The mecA gene encodes an additional penicillin-binding protein $2 \mathrm{a}(\mathrm{PBP} 2 \mathrm{a})$, which mediates cell wall synthesis in the presence of $\beta$-lactam antibiotics [6]. Together with its regulators $m e c I-m e c R 1$ and site specific recombination genes $\operatorname{cr} A$ and $c r B$, the mecA gene, is located on a mobile genetic element known as staphylococcal cassette chromosome mec (SCCmec) [7]. A number of studies have demonstrated the transfer of $m e c A$ gene from coagulase-negative staphylococcal species to $S$. aureus in vivo, and thus contributing to more successful $S$. aureus clones [8]. To date 11 SCCmec types have been reported based on combinations of mec (A, B, C1, C2 and D) and $c c r(\mathrm{AB} 1, \mathrm{AB} 2$, $\mathrm{AB} 3, \mathrm{AB} 4$ and $c \mathrm{crC}$ ) complexes and so called J regions (1, 2, 3) [9].

Traditionally recognised as hospital associated pathogens, methicillin resistant coagulase negative staphylococci (MRCoNS) have recently been linked with a range of ecological niches (community, wildlife and environmental sources) [10-12]. As a result, today increasing attention is being paid to the rapid spread of MR-CoNS and their role in transmission within the community and non - hospital settings [13].

In this study we demonstrate the dissemination of antibiotic resistance in CoNS isolated from various environmental sites in London, UK. The characterization of mecA gene and the SCCmec elements provide insights into the diversity of environmental CoNS clones.

\section{Methods}

\section{Isolation}

Four hundred seventy-nine samples were collected from different environmental sites in various locations $(n=355)$ and from the hands of volunteers $(n=124)$ in London UK between April 2013 and Nov 2014. Environmental sites included hotels $(n=100)$, baby care facilities $(n=65)$, handbags $(n=43)$, supermarkets $(n=37)$, restaurants $(n=36)$, public transport $(n=54)$, and a public library $(n=20)$. All specimens were plated on Mannitol Salt Agar (Oxoid, Basingstoke, UK), and then incubated aerobically at $37{ }^{\circ} \mathrm{C}$ for 24-72 h). One or two colonies for each site were selected based on staphylococci morphology [4]. The colonies were then purified on Nutrient Agar (Oxoid, Basingstoke, UK).

\section{Identification}

All isolates were initially screened using Gram staining, catalase and coagulase tests. Those that demonstrated potential staphylococci characteristics were identified by Matrix-assisted laser desorption ionization time flight mass-spectroscopy (MALDI-TOF-MS, Microflex LT, Bruker Daltonics, Coventry, UK) in a positive linear mode (2000-20,000 m/z range) as described previously [12]. The resulting spectra were compared with reference spectra by using the Biotyper 3.0 software (Bruker Daltonics, Coventry, UK). Escherichia. coli DH5a (Bruker Daltonics, Coventry, UK) was used as a standard for calibration and quality control.

\section{Antimicrobial susceptibility test}

A panel of 11 antibiotics was used to determine the antibiotic susceptibility of all the isolates. The standard disk diffusion method was used to test AM: amoxicillin $(10 \mu \mathrm{g})$; CEP: cefepime (30 $\mu \mathrm{g})$; CHL: chloramphenicol (30 $\mu \mathrm{g})$; ERY: erythromycin $(5 \mu \mathrm{g})$; FC: fusidic acid $(10 \mu \mathrm{g})$; GEN: gentamicin $(10 \mu \mathrm{g})$; MUP: mupirocin $(20 \mu \mathrm{g})$; OX: oxacillin $(1 \mu \mathrm{g})$; PEN: penicillin (1 unit); STR: streptomycin $(10 \mu \mathrm{g})$; TET: tetracycline $(10 \mu \mathrm{g})$;. The susceptible, intermediate resistant or resistant were determined by the Guidelines for Susceptibility Testing [14]. The Minimum Inhibitory Concentrations (MIC) for oxacillin were additionally evaluated using "M.I.C. evaluators" (Oxoid Ltd., Basingstoke, UK).

\section{Detection of mecA gene and staphylococcal cassette chromosome mec (SCCmec) typing}

The mecA gene was determined by using PCR method as described previously [15]. For mecA positive isolates, $\mathrm{SCC} m e c$ types were determined by evaluating $m e c$ and ccr complexes [15].

\section{MLST typing of Staphylococcus epidermidis}

Multi-locus sequence typing (MLST) was used to determine the sequence types of S. epidermidis [16]. Sequence types were assigned using the $S$. epidermidis database (www.mlst.net).

\section{Results}

\section{Purification of isolates}

A total of 643 staphylococci isolates were recovered in this study, including those from hotels $(n=74)$, baby care facilities $(n=46)$, handbags $(n=17)$, supermarkets $(n=89)$, restaurants $(n=96)$, public transportation $(n=94)$, human hands $(n=192)$ and public libraries $(n=35)$ (Additional file 1: Table S1). 


\section{Species determination}

Six hundred forty-three staphylococcal isolates belonging to 19 staphylococcal species were identified in this study. This included: S. epidermidis $(n=193), S$. hominis $(n=161), S$. capitis $(n=77), S$. warneri $(n=63), S$. haemolyticus $(n=45), S$. pasteuri $(n=33)$, S. saprophyticus $(n=20)$, S. aureus $(n=12)$, S. simiae $(n=10)$, S. cohnii $(n=$ 9), S. sciuri $(n=5)$, S. pettenkoferi $(n=3)$, S. auricularis $(n=$ $2)$, S. caprae $(n=2)$, S. equorum $(n=2)$, S. lugdunensis $(n=$ 2), S. xylosus $(n=2)$, S. arlettae $(n=1)$, and S. simulans ( $n$ $=1$ ). $S$. epidermidis was the predominant species, followed by S. hominis, S. capitis, S. warneri, S. haemolyticus, S. pasteuri, and S. saprophyticus However, the occurrence of the species varied for different sites. S. epidermidis was predominant among the isolates recovered from restaurants, public transport, hands and handbags, whereas $S$. hominis was predominant among the isolates recovered from supermarkets, baby care facilities and hotels and $S$. haemolyticus was predominantly isolated from the library (Table 1).

\section{Antibiotic susceptibility test results}

The disc diffusion method was used to test 606 isolates against a panel of 11 antibiotics. 572 (94\%) isolates were resistant to at least one antibiotic, and only 34 isolates were fully susceptible. Resistance to penicillin, and fusidic acid was observed in more than $65 \%$ of all staphylococcal isolates tested. 202 (33\%) isolates were resistant to streptomycin, 190 (31\%) to erythromycin, 161 (27\%) to amoxicillin, $98(16 \%)$ to tetracycline, $87(14 \%)$ to mupirocin, 59 (10\%) to gentamicin, 48 (8\%) cefepime, 36 (6\%) oxacillin, and 21(3\%) chloramphenicol (Table 2).

\section{mecA gene determination and $\mathrm{SCCmec}$ typing results}

Sixty-eight (11\%) mecA positive staphylococcal isolates were determined, however, no MRSA was determined in this study. S. sciuri had the highest mecA gene carriage

Table 1 Predominant and common staphylococcal species recovered from the human hands and different environmental sites

\begin{tabular}{lll}
\hline Sites & Predominant species (\%) & Commonly isolated species (\%) \\
\hline BCF & S. hominis (17\%) & S. warneri (17\%) \\
DSH & S. hominis (30\%) & S. haemolyticus (18\%) \\
DSL & S. haemolyticus (29\%) & S. epidermidis (26\%) \\
DSR & S. epidermidis (38\%) & S. hominis (35\%) \\
DSS & S. hominis (44\%) & S. epidermidis (29\%) \\
DST & S. epidermidis (35\%) & S. capitis (15\%) \\
HB & S. epidermidis (40\%) & S. capitis (27\%) \\
HH & S. epidermidis (36\%) & S. hominis (23\%) \\
\hline
\end{tabular}

$B C F$ baby care facilities, $D S H$ different sites of hotels, $D S L$ different sites of a library, DSR different sites of restaurants, DSS different sites of supermarkets; $D S T$ different sites of transportation facilities, $H B$ handbags, $H H$ human hand
(80\%) among all 19 staphylococcal species, followed by S. cohnii (33\%), S. haemolyticus (22\%), and S. saprothyticus $(20 \%)$. Other isolates demonstrated relatively lower carriage of mecA gene, including S.hominis (3\%), S.capitis (8\%), S. epidermidis (11\%), S.warneri (11\%), S.pasteuri (13\%). No mecA gene was found in the remaining 10 species, including S. aureus, S. simiae, S. equorum, S. caprae, S. xylosus, S. auricularis, S. simulans, S. arlettae S.pettenkoferi, and S. lugdunensis.

$\mathrm{SCC}$ mec types were fully determined in forty-six isolates. Twenty-two out of 68 isolates lacked either the mec gene complex or the $c c r$ gene complex. Thirteen staphylococci (19\%) carried SCCmec type V, followed by 8 isolates carrying SCCmec type I (2\%), 5 isolates SCCmec type IV (7\%), 4 isolates SCCmec type II (6\%), 1 isolate SCCmec type III (2\%), 1 isolate SCCmec type VI (2\%), and 1 isolate SCCmec type VIII (2\%). In addition, three isolates harboured a new SCCmec type 1A, which carried combination of class A mec complex and ccr type 1. Of the ten isolates that were non-typeable, three carried a combination of class A mec complex and $c c r C$, six carried a combination of class $\mathrm{B}$ $m e c$ and $c c r C$, and one carried class B mec and $c c r$ type 3 (Table 3).

\section{Multi-locus sequence typing of $S$. epidermidis}

MLST was performed to determine the housekeeping genes of 13 oxacillin resistant and mecA positive $S$. epidermidis. MLST typing revealed that all S. epidermidis strains possess new MLST types. MLST types of S. epidermidis isolates with in house numbers of $279,133,134$, $135,126,259,124,127,234,187,308,153$ and 191 were respectively assigned as ST599, ST600, ST600, ST600, ST601, ST602, ST602, ST603, ST604, ST605, ST606, ST607 and ST608 (Table 4). Three S. epidermidis isolates shared the same sequence types (ST), including S. epidermidis 133, 134 and 135 that were isolated from different sites of a library (DSL) possessed ST600 whereas S. epidermidis 259, and S. epidermidis 124 that had ST602 sequence type were isolated from the human hands $(\mathrm{HH})$ and different sites of hotels (DSH) respectively.

\section{Discussion}

\section{Environmental staphylococcal species}

Although antibiotic resistance is commonly linked to the clinic, recent studies from different ecological niches revealed multidrug resistant bacteria is widespread in the environment $[11,12,17]$.

We have previously reported on high levels of antibiotic resistance in staphylococci isolated from different environmental/public settings $[11,12]$. In this study we evaluated the dissemination of antibiotic resistant staphylococci recovered from a wide range of environmental settings, and characterised the carriage of the mecA gene and the diversity of SCCmec elements in these isolates. 
Table 2 Antibiotic susceptibility profile of staphylococci isolates recovered from general public settings

\begin{tabular}{|c|c|c|c|c|c|c|c|c|c|c|c|c|}
\hline \multirow[t]{2}{*}{ Isolates } & \multirow[t]{2}{*}{ No of isolates } & \multicolumn{11}{|c|}{ Resistance to a panel of 11 antibiotics (\%) } \\
\hline & & $\overline{O X}$ & PG & MUP & CEF & GM & FC & $S$ & A & $E$ & $\mathrm{~T}$ & C \\
\hline S. epidermidis & 176 & 8 & 72 & 16 & 9 & 7 & 64 & 22 & 26 & 43 & 16 & 2 \\
\hline S. hominis & 152 & 2 & 68 & 9 & 5 & 7 & 66 & 24 & 17 & 38 & 21 & 3 \\
\hline S. capitis & 73 & 4 & 58 & 15 & 4 & 1 & 60 & 47 & 23 & 14 & 10 & 5 \\
\hline S. haemolyticus & 40 & 10 & 50 & 13 & 23 & 15 & 68 & 73 & 45 & 0 & 25 & 10 \\
\hline S. warneri & 63 & 3 & 54 & 17 & 10 & 22 & 59 & 51 & 40 & 27 & 16 & 2 \\
\hline S. pasteuri & 31 & 6 & 69 & 13 & 6 & 9 & 69 & 25 & 31 & 44 & 17 & 3 \\
\hline S. saprophyticus & 20 & 15 & 90 & 25 & 5 & 10 & 100 & 10 & 25 & 35 & 15 & 10 \\
\hline S. aureus & 12 & 0 & 83 & 17 & 0 & 58 & 83 & 33 & 50 & 25 & 0 & 8 \\
\hline S. simiae & 10 & 0 & 10 & 0 & 0 & 0 & 40 & 0 & 0 & 0 & 0 & 0 \\
\hline S. cohnii & 9 & 24 & 67 & 11 & 33 & 0 & 78 & 78 & 22 & 56 & 11 & 0 \\
\hline S. sciuri & 5 & 60 & 60 & 80 & 0 & 20 & 80 & 80 & 40 & 0 & 0 & 0 \\
\hline S. pettenkoferi & 3 & 0 & 33 & 0 & 0 & 0 & 67 & 67 & 33 & 0 & 0 & 0 \\
\hline S. lugdunensis & 2 & 0 & 50 & 0 & 0 & 0 & 50 & 0 & 0 & 0 & 0 & 0 \\
\hline S. equorum & 2 & 0 & 50 & 50 & 0 & 0 & 50 & 50 & 50 & 0 & 50 & 0 \\
\hline S. caprae & 2 & 0 & 100 & 0 & 100 & 100 & 100 & 50 & 50 & 0 & 0 & 0 \\
\hline S. xylosus & 2 & 0 & 100 & 50 & 50 & 50 & 100 & 100 & 0 & 0 & 50 & 0 \\
\hline S. auricularis & 2 & 0 & 50 & 0 & 50 & 0 & 50 & 0 & 50 & 0 & 0 & 0 \\
\hline S. arlettae & 1 & 0 & 100 & 0 & 0 & 0 & 100 & 100 & 100 & 100 & 0 & 0 \\
\hline S. simulans & 1 & 0 & 100 & 0 & 0 & 0 & 100 & 0 & 0 & 0 & 0 & 0 \\
\hline
\end{tabular}

OX oxacillin $(1 \mu \mathrm{g}), P G$ penicillin $\mathrm{G}(1 \mathrm{unit}), M U P$ mupirocin $(20 \mu \mathrm{g}), C E F$ cefepime $(30 \mu \mathrm{g}), \mathrm{GM}$ gentamicin $(10 \mu \mathrm{g}), F C$ fusidic acid $(10 \mu \mathrm{g}), S$ streptomycin $(10 \mu \mathrm{g}), A$ amoxicillin $(10 \mu \mathrm{g}), E$ erythromycin $(5 \mu \mathrm{g}), T$ tetracycline $(10 \mu \mathrm{g}), C$ chloramphenicol $(30 \mu \mathrm{g})$

Six hundred and forty-three staphylococci isolates belonging to 19 species, including S. epidermidis, S. hominis, S. haemolyticus, S. capitis, S. warneri, S. pasteuri, S. saprophyticus, S. cohnii, S. aureus, S. simiae, S. sciuri, S. pettenkoferi, S. lugdunensis, S. equorum, S. caprae, S. xylosus, S. auricularis, S. simulans, and $S$. arlettae, were identified in this study. Interestingly, many of the staphylococci species recovered in our study have previously been associated with the community, preserved food, and wildlife $[4,10]$.

\section{Antibiotic resistance}

Antibiotic resistance of staphylococci associated with healthcare settings is well documented, however, little is known about the antibiotic resistance in staphylococci isolated from different ecological niches [4]. In this study, the majority of staphylococci were resistant to penicillin (65\%) and fusidic acid (66\%) (Fig. 1). Despite that $80 \%$ of hospital associated CoNS (across Europe) were reported to be resistant to oxacillin [18], only $6 \%$ of CoNS were resistant to oxacillin in this study. In addition, the levels of resistance to chloramphenicol (3\%), cefepime (8\%), gentamicin $(10 \%)$, mupirocin $(14 \%)$, tetracycline $(16 \%)$, and erythromycin (31\%) were lower compared to those reported in clinical settings [19-22]. In contrast, the rates of resistance to fusidic acid (66\%), amoxicillin (27\%) and streptomycin (33\%) in environmental staphylococcal isolates were higher than those reported in clinical staphylococci isolates $[21,23,24]$. It is widely accepted that higher levels of antibiotic resistance in clinical isolates are due to consistent antibiotic exposure [25]. The environment may also contribute to the development of antibiotic resistance in microorganisms due to human/ animal therapeutics, sewage, agriculture and industrial use of antibiotics [26]. Therefore, the wide dissemination of multidrug resistant CoNS in non-healthcare associated environments is a disturbing finding. In our study, 94\% of staphylococcal isolates were phenotypically resistant to at least 1 antibiotic, $18 \%$ were resistant to five or more antibiotics and only $6 \%$ staphylococcal isolates were fully susceptible. The study also revealed that the number of isolates resistant to multiple antibiotics varied between the different isolation sites. The least number of multiple antibiotic resistant CoNS isolates were recovered from the public transport (58\%), the highest was isolated from hotels $(78 \%)$.

\section{Methicillin-resistant staphylococci}

Methicillin resistant staphylococci pose a major public health threat, and cause severe economic and health consequences [27]. Methicillin resistance is determined by the $m e c A$ gene, which encodes for penicillin binding protein $2 \mathrm{a}$ (PBP2a) that has a low affinity to $\beta$-lactam antibiotics [28]. Hussain et al. assessed the correlation between mecA 
Table 3 Molecular characterisation and antibiotic resistance of mecA gene positive staphylococci

\begin{tabular}{|c|c|c|c|c|c|c|c|c|c|c|c|c|c|c|c|c|c|}
\hline ID & Sites & Species & $P G$ & MUP & CEF & GM & FC & $S$ & A & $E$ & $\mathrm{~T}$ & C & mecA & mec & $\mathrm{ccr}$ & sccmec & $\mathrm{MIC} / \mathrm{OX}\left(\mathrm{mg} \mathrm{I}^{-1}\right)$ \\
\hline 71 & $\mathrm{HH}$ & S. capitis & $S$ & S & $S$ & S & $S$ & $\mathrm{R}$ & $S$ & $S$ & $S$ & $S$ & + & - & - & I & 0.5 \\
\hline 100 & DSH & S. cohnii & $\mathrm{R}$ & $\mathrm{R}$ & S & S & S & $S$ & $\mathrm{R}$ & $\mathrm{R}$ & S & S & + & Class A & 5 & $5 A$ & 1 \\
\hline 97 & BCF & S. cohnii & $\mathrm{R}$ & S & $\mathrm{R}$ & S & $\mathrm{R}$ & $\mathrm{R}$ & $S$ & $\mathrm{R}$ & $\mathrm{S}$ & S & + & Class B & 1 & I & 0.25 \\
\hline 279 & $\mathrm{HH}$ & S. epidermidis & $\mathrm{R}$ & S & S & S & $\mathrm{R}$ & $S$ & $S$ & $\mathrm{R}$ & S & S & + & Class B & 2 & IV & 2 \\
\hline 127 & DSH & S. epidermidis & $\mathrm{R}$ & S & I & S & S & $S$ & $\mathrm{R}$ & $\mathrm{R}$ & $\mathrm{R}$ & S & + & Class C & 5 & V & 2 \\
\hline 139 & DSR & S. epidermidis & $\mathrm{R}$ & $\mathrm{R}$ & $\mathrm{R}$ & S & $\mathrm{R}$ & S & $\mathrm{R}$ & $\mathrm{R}$ & $\mathrm{R}$ & S & + & Class C & 5 & V & 2 \\
\hline 191 & DSS & S. epidermidis & $\mathrm{R}$ & S & S & S & R & $S$ & $\mathrm{R}$ & $S$ & $S$ & $S$ & + & Class B & 4 & $\mathrm{Vl}$ & 2 \\
\hline 153 & DSH & S. epidermidis & $\mathrm{R}$ & S & S & S & $\mathrm{R}$ & S & S & S & S & $S$ & + & Class C & 5 & V & 1 \\
\hline 187 & DSS & S. epidermidis & $\mathrm{R}$ & $S$ & S & S & S & $S$ & $S$ & $\mathrm{R}$ & S & S & + & Class C & 5 & V & 1 \\
\hline 134 & DSL & S. epidermidis* & $\mathrm{R}$ & S & S & S & $\mathrm{R}$ & $\mathrm{R}$ & $\mathrm{R}$ & $\mathrm{R}$ & $\mathrm{R}$ & $S$ & + & Class B & 1 & I & 1 \\
\hline 259 & $\mathrm{HH}$ & S. epidermidis & $\mathrm{R}$ & $S$ & $\mathrm{R}$ & S & $\mathrm{R}$ & $\mathrm{R}$ & $\mathrm{R}$ & $\mathrm{R}$ & S & S & + & Class C & 5 & V & 1 \\
\hline 135 & DSL & S. epidermidis* & $\mathrm{R}$ & S & S & S & $\mathrm{R}$ & $\mathrm{R}$ & $\mathrm{R}$ & $\mathrm{R}$ & $\mathrm{R}$ & S & + & Class B & 2 & IV & 0.5 \\
\hline 124 & DSH & S. epidermidis & $\mathrm{R}$ & S & $\mathrm{R}$ & S & $\mathrm{R}$ & $\mathrm{R}$ & $\mathrm{R}$ & S & $\mathrm{R}$ & S & + & Class B & 2 & IV & 0.5 \\
\hline 133 & DSL & S. epidermidis* & $\mathrm{R}$ & S & S & S & $\mathrm{R}$ & $\mathrm{R}$ & $\mathrm{R}$ & R & $\mathrm{R}$ & S & + & Class B & 3 & $3 B$ & 0.5 \\
\hline 126 & $\mathrm{HH}$ & S. epidermidis & $\mathrm{R}$ & S & I & S & S & $\mathrm{R}$ & $\mathrm{R}$ & $\mathrm{R}$ & $\mathrm{S}$ & $\mathrm{S}$ & + & - & - & III & 0.5 \\
\hline 119 & DSH & S. epidermidis & $\mathrm{R}$ & S & S & S & S & $S$ & $\mathrm{R}$ & I & $\mathrm{R}$ & S & + & Class C & 5 & V & 0.12 \\
\hline 111 & BCF & S. epidermidis & $\mathrm{R}$ & S & S & S & S & S & S & S & S & S & + & Class A & 2 & $\|$ & 0.12 \\
\hline 202 & DST & S. epidermidis & S & $\mathrm{R}$ & S & S & $\mathrm{R}$ & । & $S$ & S & S & S & + & Class B & 5 & $5 B$ & 0.12 \\
\hline 264 & $\mathrm{HH}$ & S. epidermidis & S & $\mathrm{R}$ & S & S & $\mathrm{R}$ & $\mathrm{R}$ & S & S & $S$ & S & + & Class B & 2 & IV & 0.06 \\
\hline 129 & DSL & S. epidermidis & $\mathrm{R}$ & S & S & S & $\mathrm{R}$ & $\mathrm{R}$ & $\mathrm{R}$ & $\mathrm{R}$ & $\mathrm{R}$ & S & + & Class B & 1 & I & 0.03 \\
\hline 362 & DSL & S. haemolyticus & $\mathrm{R}$ & S & 1 & S & S & R & $\mathrm{R}$ & S & $S$ & S & + & Class C & 5 & V & 2 \\
\hline 367 & DSL & S. haemolyticus & $\mathrm{R}$ & S & $\mathrm{R}$ & S & $\mathrm{S}$ & $\mathrm{R}$ & $\mathrm{R}$ & S & $\mathrm{S}$ & $S$ & + & Class C & 5 & V & 2 \\
\hline 355 & DSH & S. haemolyticus & $\mathrm{R}$ & S & $\mathrm{R}$ & $\mathrm{R}$ & $\mathrm{R}$ & $\mathrm{R}$ & $\mathrm{R}$ & S & $\mathrm{R}$ & S & + & Class C & 5 & V & 2 \\
\hline 384 & $\mathrm{HH}$ & S. haemolyticus & $\mathrm{R}$ & $\mathrm{R}$ & S & S & $\mathrm{R}$ & $\mathrm{R}$ & $S$ & S & $\mathrm{S}$ & $S$ & + & Class C & 5 & V & 2 \\
\hline 322 & DSH & S. haemolyticus & $\mathrm{R}$ & S & S & S & S & 1 & $\mathrm{R}$ & S & $\mathrm{R}$ & S & + & Class A & 1 & $1 \mathrm{~A}$ & 0.25 \\
\hline 382 & $\mathrm{HH}$ & S. haemolyticus & $\mathrm{R}$ & S & I & S & $\mathrm{R}$ & $\mathrm{R}$ & S & $\mathrm{R}$ & S & S & + & - & - & $\|$ & 0.25 \\
\hline 323 & DSH & S. haemolyticus & S & S & S & $\mathrm{R}$ & S & $\mathrm{R}$ & $\mathrm{R}$ & I & $\mathrm{R}$ & S & + & Class A & 2 & $\|$ & 0.12 \\
\hline 381 & $\mathrm{HH}$ & S. haemolyticus & S & S & I & S & S & $\mathrm{R}$ & S & S & S & S & + & Class B & 5 & $5 B$ & 0.12 \\
\hline 360 & DSH & S. haemolyticus & S & S & S & S & $\mathrm{R}$ & $S$ & S & S & $\mathrm{S}$ & $\mathrm{R}$ & + & Class B & 5 & $5 B$ & 0.06 \\
\hline 369 & DSL & S. haemolyticus & $\mathrm{R}$ & S & S & S & $\mathrm{R}$ & $\mathrm{R}$ & S & $\mathrm{R}$ & S & $\mathrm{R}$ & + & Class B & 1 & I & 0.03 \\
\hline 413 & DSH & S. hominis & S & $\mathrm{R}$ & S & S & $\mathrm{R}$ & $\mathrm{R}$ & S & S & $\mathrm{S}$ & $S$ & + & Class C & 5 & V & 2 \\
\hline 506 & DSS & S. hominis & S & S & S & S & R & S & S & S & S & S & + & Class B & 1 & I & 0.5 \\
\hline 400 & DSH & S. hominis & $\mathrm{R}$ & $\mathrm{R}$ & S & S & $\mathrm{R}$ & S & $\mathrm{R}$ & $\mathrm{R}$ & $\mathrm{R}$ & $S$ & + & Class A & 1 & $1 \mathrm{~A}$ & 0.12 \\
\hline 326 & DSH & S. hominis & S & S & S & S & S & S & $\mathrm{R}$ & 1 & S & S & + & Class A & 1 & $1 \mathrm{~A}$ & 0.06 \\
\hline 589 & $\mathrm{HB}$ & S. pasteuri & $\mathrm{R}$ & S & S & S & $\mathrm{R}$ & $\mathrm{R}$ & $S$ & $\mathrm{R}$ & $S$ & $S$ & + & Class A & 5 & $5 \mathrm{~A}$ & 0.25 \\
\hline 592 & $\mathrm{HH}$ & S. pasteuri & S & $\mathrm{R}$ & S & S & $\mathrm{R}$ & $\mathrm{R}$ & $S$ & $S$ & $\mathrm{~S}$ & $S$ & + & Class B & 5 & $5 B$ & 0.25 \\
\hline 627 & $\mathrm{HH}$ & S. saprophyticus & $\mathrm{R}$ & I & S & S & R & $S$ & S & S & $\mathrm{R}$ & $\mathrm{S}$ & + & Class B & 5 & $5 B$ & 0.5 \\
\hline 621 & DSS & S. saprophyticus & $\mathrm{R}$ & $\mathrm{R}$ & $\mathrm{R}$ & S & $\mathrm{R}$ & $S$ & $\mathrm{R}$ & $S$ & $\mathrm{R}$ & $S$ & + & Class B & 2 & IV & 0.25 \\
\hline 630 & $\mathrm{HH}$ & S. sciuri & $\mathrm{R}$ & $\mathrm{R}$ & 1 & S & $\mathrm{R}$ & $\mathrm{R}$ & $S$ & S & $S$ & $S$ & + & Class A & 4 & VIII & 2 \\
\hline 632 & DSH & S. sciuri & $\mathrm{R}$ & S & I & S & $\mathrm{R}$ & $\mathrm{R}$ & $\mathrm{R}$ & S & $\mathrm{S}$ & $S$ & + & Class A & 5 & $5 \mathrm{~A}$ & 1 \\
\hline 633 & DSH & S. sciuri & $\mathrm{R}$ & $\mathrm{R}$ & I & $\mathrm{R}$ & $\mathrm{R}$ & $\mathrm{R}$ & $\mathrm{R}$ & $\mathrm{S}$ & $\mathrm{S}$ & $S$ & + & Class B & 5 & $5 B$ & 1 \\
\hline 629 & $\mathrm{HH}$ & S. sciuri & S & $\mathrm{R}$ & S & $S$ & S & $S$ & $S$ & S & $\mathrm{S}$ & $\mathrm{S}$ & + & - & - & $\|$ & 0.25 \\
\hline 704 & $\mathrm{HH}$ & S. warneri & $\mathrm{R}$ & S & 1 & S & R & $\mathrm{R}$ & $\mathrm{R}$ & $\mathrm{R}$ & $\mathrm{S}$ & $S$ & + & Class C & 5 & V & 0.5 \\
\hline 662 & DSH & S. warneri & $\mathrm{R}$ & S & S & $\mathrm{R}$ & $\mathrm{R}$ & $\mathrm{R}$ & $\mathrm{R}$ & S & $\mathrm{R}$ & S & + & Class C & 5 & V & 0.25 \\
\hline
\end{tabular}


Table 3 Molecular characterisation and antibiotic resistance of mecA gene positive staphylococci (Continued)

\begin{tabular}{lllllllllllllllllll}
\hline ID & Sites & Species & PG & MUP & CEF & GM & FC & S & A & E & T & C & mecA & mec & ccr & SCCmec & MIC/OX (mg I $\left.)^{-1}\right)$ \\
\hline 694 & HH & S. warneri & R & S & S & S & S & S & S & S & R & S & + & - & & - & I & $\mathbf{0 . 2 5}$ \\
655 & BCF & S. warneri & S & R & S & R & R & R & S & I & S & S & + & Class B & 1 & I & $\mathbf{0 . 1 2}$ &
\end{tabular}

Note: * S. epidermidis isolates with similar MLST types

$R$ : resistant, $S$ sensitive. I intermediate

$B C F$ baby care facility, DSH different sites of hotels, DSL different sites of a library, DSR different sites of restaurants, DSS different sites of supermarkets, DST different sites of transportation facilities, $H B$ handbags, $H H$ human hands

A amoxicillin $(10 \mu \mathrm{g}), C E F$ cefepime $(30 \mu \mathrm{g}), C$ chloramphenicol $(30 \mu \mathrm{g}), E$ erythromycin $(5 \mu \mathrm{g}), F C$ fusidic acid $(10 \mu \mathrm{g}), \mathrm{GM}$ gentamicin $(10 \mu \mathrm{g}), M U P$ mupirocin $(20 \mu \mathrm{g}), O X$ oxacillin $(1 \mu \mathrm{g}), P G$ penicillin $\mathrm{G}(1 \mathrm{unit}), \mathrm{S}$ streptomycin $(10 \mu \mathrm{g}), T$ tetracycline $(10 \mu \mathrm{g})$

gene and oxacillin susceptibility breakpoints $\left(0.5 \mathrm{mg} \mathrm{l}^{-1}\right)$ of 493 clinical CoNS belonging to and classified into 4 categories [29]. The mecA gene positive staphylococci were categorized into groups I and II, and demonstrated that group I (S. haemolyticus (83.3\%), S. epidermidis (61.9\%), S. hominis (51.8\%)) differs from group II (S. cohnii (28.5\%), S. warneri (27.3\%), S. saprophyticus (9.0\%)) by their high levels of mecA-carriage [29]. Interestingly, S. hominis (38\%), S. haemolyticus (22\%), and S. epidermidis (7\%) isolated in this study harboured significantly lower levels of the mecA gene. Moreover, in this study S. cohnii (33\%) and S. saprophyticus (10\%) showed higher mecA gene carriage than clinical isolates reported by Hussain, et al. [29], whereas the levels of $m e c A$ gene carriage in $S$. warneri (6\%) were lower than in clinical isolates. No mecA gene was detected in staphylococcal species of groups III and IV, which included S. xylosus, S. lugdunensis, S. capitis, S.simulans, and S. schleiferi [29]. Similarly, in this study S. lugdunensis, S. xylosus and $S$. simulans were determined to be susceptible to oxacillin and lacked mecA gene. However, in contrast to the reports by Hussain, et al. [29] we found that $m e c A$ gene was present in $8 \%$ of $S$. capitis isolates.

Oxacillin susceptible mecA gene positive $S$. aureus (OSMRSA) has been reported worldwide, and the risk of induced high levels of oxacillin resistance was determined in OS-MRSA [30, 31]. In this study, 68 (46\%) staphylococcal isolates were confirmed by PCR to carry the mecA gene, however, they were phenotypically susceptible to oxacillin with the MICs (oxacillin) varying from 0.015 to $2 \mathrm{mg} \mathrm{l}^{-1}$. This study demonstrates the prevalence of mecA positive but oxacillin susceptible CoNS (OS-CoNS) in the environment. Little is known about OS-CoNS isolates recovered from the environment and their epidemiological data are limited. Additional studies are necessary to further our understanding of the prevalence and molecular epidemiology of OS-CoNS in the environment.

\section{SCCmec elements}

SCCmec is a mobile genetic element with two essential components: the mec gene complex, and the cassette chromosome recombinase ( $c c r)$ gene complex [32]. The combination of the mec gene complex and $c \mathrm{cr}$ gene complex confers different SCCmec types [32]. SCCmec type I, II, III are reported to be associated with MRSA recovered from healthcare settings, whereas SCCmec type IV and V are mainly associated with the community [32]. Moreover, it has been shown that the size of SCCmec types IV and V are smaller than SCCmec types I, II and III, thus conferring

Table 4 MLST types of 13 oxacillin resistant and mecA positive S. epidermidis

\begin{tabular}{lllllllllll}
\hline ID & Sites & Species & arCC & aroE & gtr & mutS & pyrR & tpiA & yqiL & MLST types \\
\hline 279 & HH & S. epidermidis & 57 & 17 & 5 & 5 & 3 & 4 & 31 & ST599 \\
133 & DSL & S. epidermidis & 57 & 1 & 2 & 2 & 4 & 1 & 4 & ST600 \\
134 & DSL & S. epidermidis & 57 & 1 & 2 & 2 & 4 & 1 & 4 & ST600 \\
135 & DSL & S. epidermidis & 57 & 1 & 2 & 2 & 4 & 1 & 4 & ST600 \\
126 & HH & S. epidermidis & 57 & 25 & 9 & 5 & 6 & 1 & 8 & ST601 \\
259 & HH & S. epidermidis & 57 & 1 & 2 & 2 & 4 & 1 & 1 & ST602 \\
124 & DSH & S. epidermidis & 57 & 1 & 2 & 2 & 4 & 1 & 1 & ST602 \\
127 & DSH & S. epidermidis & 57 & 10 & 5 & 5 & 10 & 16 & 21 & ST603 \\
234 & HB & S. epidermidis & 57 & 1 & 1 & 1 & 2 & 41 & 1 & ST604 \\
187 & DSS & S. epidermidis & 57 & 1 & 1 & 2 & 2 & 1 & 1 & ST605 \\
308 & HH & S. epidermidis & 57 & 1 & 2 & 2 & 4 & 7 & 1 & ST606 \\
153 & DSH & S. epidermidis & 57 & 1 & 22 & 2 & 2 & 16 & 1 & ST607 \\
191 & DSS & S. epidermidis & 57 & 3 & 5 & 5 & 7 & 14 & 11 & ST608
\end{tabular}

$H H$ human hands, DSL different sites of a library, DSH different sites of hotels, DSS different sites of supermarkets MLST Multi-locus sequence typing 


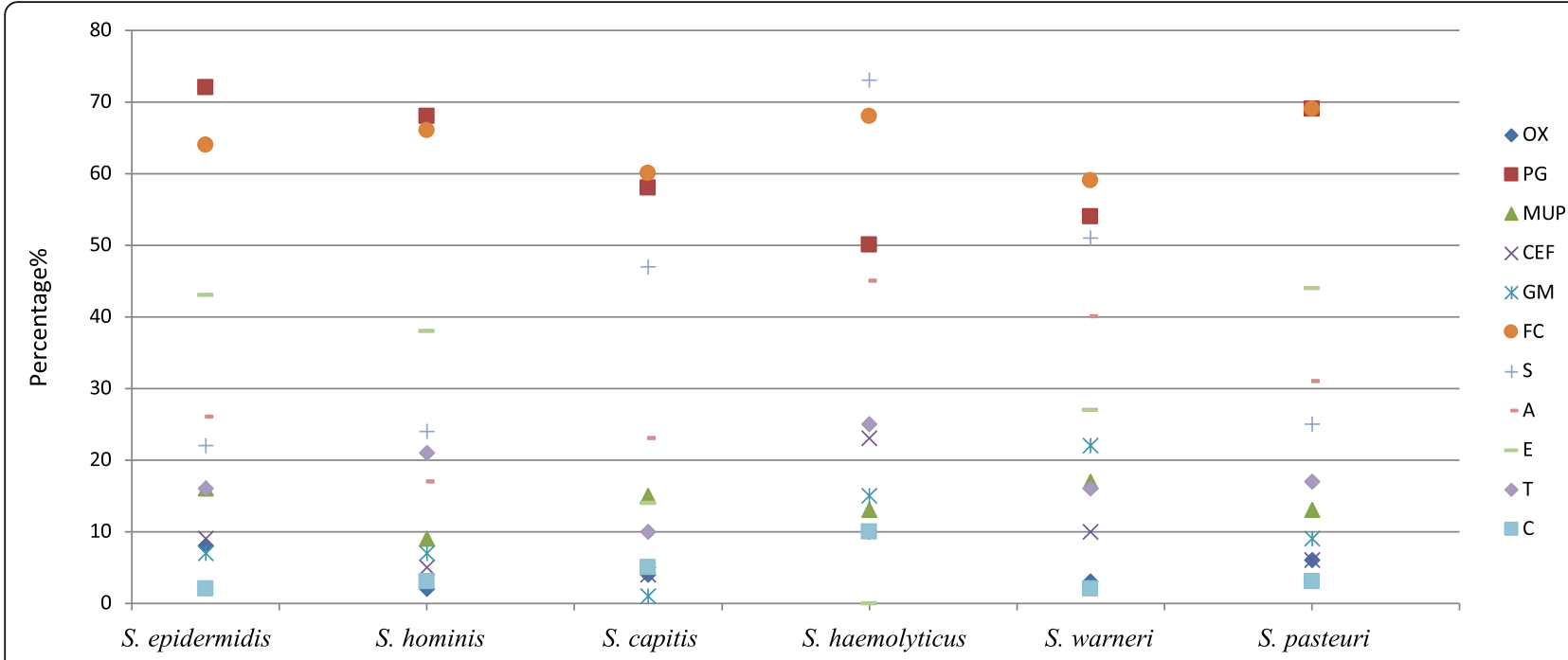

Fig. 1 The scatter plot of staphylococcal (> 30 isolates) susceptibility profile. Legend: OX: oxacillin ( $1 \mu \mathrm{g})$; PG: penicillin G (1 unit); MUP: mupirocin $(20 \mu \mathrm{g})$; CEF: cefepime $(30 \mu \mathrm{g})$; GM: gentamicin $(10 \mu \mathrm{g})$; FC: fusidic acid $(10 \mu \mathrm{g})$; S: streptomycin $(10 \mu \mathrm{g}) ; \mathrm{A}$ : amoxicillin (10 $\mu \mathrm{g})$; E: erythromycin $(5 \mu \mathrm{g}) ; \mathrm{T}$ : tetracycline $(10 \mu \mathrm{g}) ; \mathrm{C}$ : chloramphenicol $(30 \mu \mathrm{g})$

increased mobility by their smaller size and contributing the spread of these smaller SCCmec elements [33]. In this study, SCCmec type I, II or III were found in $19 \%(n=13)$ of mecA-positive CoNS, whereas $27 \%(n=18)$ of CoNS were determined to harbour SCCmec type IV or V. SCCmec type VI and VIII were previously identified in Portugal (2006) and Canada (2009) in hospital associated MRSA (HA-MRSA) [33, 34]. In this study, we identified one of each type, however, we did not detect SCCmec types IX.

Becker et al., have previously summarized the community and livestock associated staphylococcal species and their SCCmec types, which included S. capitis (I, IA, II, III, IV, IVa, V, non-typeable: (NT)), S. cohnii (NT), S. epidermidis (I, IIa, IIb, III, III (variant), IV, IVa, IVb, IVc, IVd, IVe, IVg, V, VI, NT), S. haemolyticus (I, II, II.1, III, III (variant), IV, V, NT), S. honomis (I, III, IV, NT), S. pasteuri (IVc), S. saprophyticus (III, NT), S. sciuri (I, III, IIIA, V, VII, NT) and $S$. warneri (IV, IV.1, IVb, IVE) [4]. In this study, species associated SCCmec types differed and included the following: S. capitis (I, NT), S. haemolyticus (I, II, V, NT) and S. hominis (I, V, NT), S. cohnii (I, V, NT), S. pasteuri (NT), S. saprophyticus (IV, NT), S. sciuri (II, VIII), S. warneri (I, V, NT). S. epidermidis possessed SCCmec types similar to those reported previously [4].

Thirteen unclassified SCCmec types were determined in this study, including three carrying class A mec complex and $c c r C$, six had a combination of class B mec and $c c r C$, one carried class B mec and $c c r 3$, and three had a combination of class A mec complex and $c c r$ type 1 . The $1 \mathrm{~A}$ was previously defined as a new SCCmec type 1A by others [35]. Pseudo $(\psi)$-SCCmec harbours the mec complex but lacks $c c r$, while, SCCmec12263 is reported to carry the $c c r$ complex but lacks mec complex [36, 37]. In this study, 21 isolates $(29 \%)$ were categorized as $(\psi)$-SCCmec and SCCmec12263 since they lacked either mec complex or ccr complexes. $\psi$ SCC element is characterized by lacking genes for $c c r$ and mec [4]. One of S. saprophyticus isolates in this study was found to possess the $\psi$ SCC element (Table 5).

\section{MLST of S. epidermidis}

Whilst many studies have reported on the changing epidemiology of $S$. aureus, epidemiological data of other staphylococcal species are limited [38, 39]. In this study, 10 new MLST types were determined in 13 S. epidermidis isolates. Interestingly, although isolates recovered from human hands (S. epidermidis 259/ SCCmec V) and hotels (S. epidermidis 124/ SCCmec IV) harboured different SCCmec types, they shared the same MLST type ST602. In addition, three S. epidermidis isolates recovered from libraries (S. epidermidis 133, S. epidermidis 134, S. epidermidis 135) shared the same MLST type ST600 (Table 4). However, despite sharing the same MLST type S. epidermidis 133, S. epidermidis 134 and S. epidermidis 135 harbored SCCmec type 3B, I, IV respectively. Others reported that $S$. epidermidis ST2 was associated with type II, III, IV and non-typable SCCmec, and S. epidermidis ST22 harboured SCCmec type III, IV and V [40].

\section{Conclusions}

Systematic analysis of staphylococci isolated from non-healthcare environments provided insights into the diversity and antibiotic susceptibility patterns of these 
Table 5 The diversity of SCCmec types of mecA gene positive staphylococci

\begin{tabular}{|c|c|c|c|c|c|c|c|c|c|c|c|c|c|c|c|c|c|}
\hline ID & Sites & Species & $P G$ & MUP & CEF & GM & FC & S & A & E & T & C & mecA & mec & $\mathrm{CCr}$ & sccmec & $\operatorname{MIC/OX}\left(\mathrm{mg} \mathrm{l}^{-1}\right)$ \\
\hline 75 & $\mathrm{HH}$ & S. capitis & $R$ & $S$ & $S$ & $S$ & $R$ & $R$ & $S$ & $S$ & $S$ & $S$ & + & Class A & NT & Pseudo $(\psi)$-SCCmec & 0.5 \\
\hline 81 & $\mathrm{HH}$ & S. capitis & $\mathrm{R}$ & S & $\mathrm{R}$ & S & $\mathrm{R}$ & $\mathrm{R}$ & $\mathrm{R}$ & $\mathrm{R}$ & S & S & + & NT & 5 & scCmec12263 & 0.5 \\
\hline 70 & $\mathrm{HH}$ & S. capitis & $\mathrm{R}$ & S & S & S & S & R & S & S & $\mathrm{R}$ & S & + & NT & 5 & SCCmec12263 & 0.25 \\
\hline 83 & $\mathrm{HH}$ & S. capitis & S & $\mathrm{R}$ & S & S & $\mathrm{R}$ & $\mathrm{R}$ & S & S & $S$ & S & + & NT & 5 & scCmec12263 & 0.12 \\
\hline 24 & DSH & S. capitis & $S$ & S & S & S & $R$ & $\mathrm{R}$ & S & S & $S$ & S & + & NT & 1 & SCCmec12263 & 0.12 \\
\hline 108 & $\mathrm{HH}$ & S. cohnii & S & S & । & S & $\mathrm{R}$ & $\mathrm{R}$ & S & $\mathrm{R}$ & R & S & + & Class A & NT & Pseudo $(\psi)$-SCCmec & 1 \\
\hline 308 & $\mathrm{HH}$ & S. epidermidis & $\mathrm{R}$ & $\mathrm{R}$ & S & S & $R$ & S & $\mathrm{R}$ & $\mathrm{R}$ & $S$ & S & + & Class B & NT & Pseudo $(\psi)$-SCCmec & 2 \\
\hline 234 & $H B$ & S. epidermidis & S & $\mathrm{R}$ & S & S & $R$ & $\mathrm{R}$ & S & $R$ & $\mathrm{R}$ & S & + & Class A & NT & Pseudo $(\psi)$-SCCmec & 1 \\
\hline 249 & DSH & S. epidermidis & $\mathrm{R}$ & S & S & S & $R$ & R & S & S & $\mathrm{R}$ & S & + & NT & 2 & scCmec12263 & 0.12 \\
\hline 125 & DSH & S. epidermidis & S & S & । & S & S & $\mathrm{R}$ & S & S & S & S & + & NT & 5 & sCCmec12263 & 0.06 \\
\hline 185 & DSS & S. epidermidis & $\mathrm{R}$ & S & S & $S$ & S & S & S & S & S & S & + & Class C & NT & Pseudo $(\psi)$-SCCmec & 0.06 \\
\hline 498 & DSS & S. hominis & $\mathrm{R}$ & S & S & S & $\mathrm{R}$ & S & S & $\mathrm{R}$ & S & S & + & Class A & NT & Pseudo $(\psi)$-SCCmec & 0.5 \\
\hline 426 & DSH & S. hominis & $\mathrm{R}$ & S & I & S & $R$ & $\mathrm{R}$ & $\mathrm{R}$ & R & S & S & + & Class A & NT & Pseudo $(\psi)$-SCCmec & 0.25 \\
\hline 412 & DSH & S. hominis & $\mathrm{R}$ & S & S & S & $\mathrm{R}$ & S & $\mathrm{R}$ & $\mathrm{R}$ & S & S & + & NT & 1 & sCCmec12263 & 0.06 \\
\hline 391 & BCF & S. hominis & $\mathrm{R}$ & S & S & S & $R$ & S & S & S & $S$ & $S$ & + & NT & 5 & scCmec12263 & 0.03 \\
\hline 593 & $\mathrm{HH}$ & S. pasteuri & $\mathrm{R}$ & S & S & $\mathrm{R}$ & $R$ & $\mathrm{R}$ & $\mathrm{R}$ & S & S & S & + & NT & 5 & scCmec12263 & 0.5 \\
\hline 597 & $\mathrm{HH}$ & S. pasteuri & $\mathrm{R}$ & $\mathrm{R}$ & । & S & S & $\mathrm{R}$ & S & S & S & S & + & NT & 5 & scCmec12263 & 0.5 \\
\hline 616 & BCF & S. saprophyticus & $\mathrm{R}$ & $\mathrm{R}$ & S & S & $\mathrm{R}$ & । & $R$ & $\mathrm{R}$ & R & S & + & NT & 5 & SCCmec12263 & 256 \\
\hline 612 & BCF & S. saprophyticus & $R$ & $\mathrm{R}$ & S & S & $R$ & S & S & $\mathrm{R}$ & S & S & + & NT & NT & $\psi S C C$ & 1 \\
\hline 659 & DSH & S. warneri & R & $R$ & S & S & R & R & R & S & $S$ & S & + & NT & 5 & SCCmec12263 & 0.5 \\
\hline 648 & BCF & S. warneri & $\mathrm{R}$ & S & S & $\mathrm{R}$ & $R$ & S & $\mathrm{R}$ & S & $S$ & S & + & NT & 5 & scCmec12263 & 0.06 \\
\hline 645 & BCF & S. warneri & $\mathrm{R}$ & S & S & S & $\mathrm{R}$ & S & S & S & S & S & + & NT & 4 & scCmec12263 & 0.015 \\
\hline
\end{tabular}

$R$ resistant, $S$ sensitive, $I$ intermediate

$B C F$ baby care facility, DSH different sites of hotels, DSL different sites of a library, DSR different sites of restaurants, DSS different sites of supermarkets; DST different sites of transportation facilities, $H B$ handbags, $H H$ human hands

$A$ amoxicillin $(10 \mu \mathrm{g}), C E F$ cefepime $(30 \mu \mathrm{g}), C$ chloramphenicol $(30 \mu \mathrm{g}), E$ erythromycin $(5 \mu \mathrm{g}), F C$ fusidic acid $(10 \mu \mathrm{g}), \mathrm{GM}$ gentamicin $(10 \mu \mathrm{g}), \mathrm{MUP}$ mupirocin $(20 \mu \mathrm{g}), O X$ oxacillin $(1 \mu \mathrm{g}), P G$ penicillin $\mathrm{G}(1 \mathrm{unit}), S$ streptomycin $(10 \mu \mathrm{g}), T$ tetracycline $(10 \mu \mathrm{g})$

isolates. Multi-drug resistance was commonly seen in each staphylococcal species. The prevalence of multiple antibiotic resistant staphylococci in this study provides evidence that antibiotics in the natural environments can contribute to the selection of antibiotic resistance in microorganisms. The finding of various SCCmec types in non-healthcare associated environments emphasizes the complexity of SCCmec elements. In addition to this, we also report on new MLST types that were assigned for all S. epidermidis isolates. This highlights the genetic variability of these isolates. In conclusion, the non-healthcare environments may act as a reservoir of multidrug resistant staphylococci, and current infection control measures are ineffective in limiting the spread of these bacteria.

\section{Additional file}

Additional file 1: Table S1. Isolates collected from different environmental sites and human hands (PDF $46 \mathrm{~kb}$ )

\section{Abbreviations}

AM: Amoxicillin; BCF: Baby care facility; CEP: Cefepime;

CHL: Chloramphenicol; CoNS: Coagulase-negative staphylococci; DSH: Different sites of hotels; DSL: Different sites of a library; DSR: Different sites of restaurants; DSS: Different sites of supermarkets; DST: Different sites of transportation facilities; ERY: Erythromycin; FC: Fusidic acid;

GEN: Gentamicin; HB: Handbags; HH: Human hands; MALDI-TOF-MS: Matrixassisted laser desorption ionization time flight mass-spectroscopy;

MIC: Minimum Inhibitory Concentrations; MLST: Multi-locus sequence typing; MR-CoNS: Methicillin resistant coagulase negative staphylococci;

MRSA: Methicillin resistant Staphylococcus aureus; MUP: Mupirocin;

OX: Oxacillin; PEN: Penicillin; SCCmec: Staphylococcal cassette chromosome mec; ST: Sequence types; STR: Streptomycin; TET: Tetracycline

\section{Funding}

This work was part of Zhen Xu's PhD study funded by China Scholarship Council.

Availability of data and materials

All data generated or analysed during this study are included in this published article and its supplementary information files.

\section{Authors' contributions}

ZX: samples collection, laboratory work, data analysis, manuscript preparation. HS: study design, critically reviewing the paper. RM: Data analysis, critically reviewing the paper. JC: data analysis, critically reviewing the paper. WZ: data analysis, critically reviewing the paper. YL: data analysis, 
critically reviewing the paper. RRC: conception and design of the study. HVM: conception and design of the study; data analysis; writing and critically reviewing the paper. All authors read and approved the final manuscript.

\section{Ethics approval and consent to participate} Not applicable.

\section{Consent for publication}

Not applicable.

\section{Competing interests}

The authors declare that they have no competing interests.

\section{Publisher's Note}

Springer Nature remains neutral with regard to jurisdictional claims in published maps and institutional affiliations.

\section{Author details}

'National Demonstration Center for Experimental Preventive Medicine Education, Tianjin Medical University, Qixiang Road No. 22, Tianjin 300070 China. ${ }^{2}$ School of Biological and Chemical Sciences, Queen Mary University of London, London, UK. ${ }^{3}$ Department of Natural Sciences, Middlesex University, The Burroughs Hendon, Middlesex NW4 4BT, UK. ${ }^{4}$ Natural History Museum, Cromwell Rd, London SW7 5BD, UK. ${ }^{5}$ Department of Infectious Diseases, Huashan Hospital, Fudan University, Shanghai 200040, China. ${ }^{6}$ Tianjin Xiqing hospital, Tianjin 300380, China. ${ }^{7}$ School of Health, Sport and Biosciences, University of East London, E1 4NS, London, UK.

Received: 19 April 2018 Accepted: 6 June 2018

Published online: 13 June 2018

\section{References}

1. Kloos WE, Bannerman TL. Update on clinical significance of coagulasenegative staphylococci. Clin Microbiol Rev. 1994;7(1):117-40.

2. Foster TJ, Geoghegan JA, Ganesh VK, Höök M. Adhesion, invasion and evasion: the many functions of the surface proteins of Staphylococcus aureus. Nat Rev Microbiol. 2014;12(1):49-62.

3. Bouchami O, Achour W, Mekni MA, Rolo J, Ben HA. Antibiotic resistance and molecular characterization of clinical isolates of methicillin-resistant coagulase-negative staphylococci isolated from bacteremic patients in oncohematology. Folia Microbiol. 2011;56(2):122-30.

4. Becker K, Heilmann C, Peters G. Coagulase-negative staphylococci. Clin Microbiol Rev. 2014;27(4):870-926.

5. Ubukata K, Nonoguchi R, Song MD, Matsuhashi M, Konno M. Homology of mecA gene in methicillin-resistant Staphylococcus haemolyticus and Staphylococcus simulans to that of Staphylococcus aureus. Antimicrob Agents Chemother. 1990;34(1):170-2.

6. Pinho MG, de Lencastre $H$, Tomasz $A$. An acquired and a native penicillinbinding protein cooperate in building the cell wall of drug-resistant staphylococci. Proc Natl Acad Sci. 2001;98(19):10886-91.

7. Hiramatsu K, Cui L, Kuroda M, Ito T. The emergence and evolution of methicillin-resistant Staphylococcus aureus. Trends Microbiol. 2001;9(10):486-93.

8. Harrison EM, Paterson GK, Holden MTG, Ba X, Rolo J, Morgan FJE, et al. A novel hybrid SCCmec-mecC region in Staphylococcus sciuri. J Antimicrob Chemother. 2014;69(4):911-8.

9. IWG-SCC. Classification of staphylococcal cassette chromosome mec (SCCmec): guidelines for reporting novel SCCmec elements. Antimicrob Agents Chemother. 2009;53(12):4961-7.

10. Pantucek R. Staphylococcus simiae sp. nov., isolated from south American squirrel monkeys. Int J Syst Evol Microbiol. 2005;55(5):1953-8.

11. Xu Z, Mkrtchyan HV, Cutler RR. Antibiotic resistance and mecA characterization of coagulase-negative staphylococci isolated from three hotels in London, UK. Front Microbiol. 2015;6:947.

12. Mkrtchyan HV, Russell CA, Wang N, Cutler RR. Could public restrooms be an environment for bacterial Resistomes? PLoS One. 2013;8(1):e54223.

13. Boyce JM. Environmental contamination makes an important contribution to hospital infection. J Hosp Infect. 2007;65(Suppl 2):50-4.

14. Andrews JM, Howe RA. BSAC standardized disc susceptibility testing method (version 10). J Antimicrob Chemother. 2011;66:2726-57.

15. Kondo Y, Ito T, Ma XX, Watanabe S, Kreiswirth BN, Etienne J, et al. Combination of multiplex PCRs for staphylococcal cassette chromosome mec type assignment: rapid identification system for mec, ccr, and major differences in junkyard regions. Antimicrob Agents Chemother. 2007;51(1):264-74.

16. Thomas JC, Vargas MR, Miragaia M, Peacock SJ, Archer GL, Enright MC Improved multilocus sequence typing scheme for Staphylococcus epidermidis. J Clin Microbiol. 2007:45(2):616-9.

17. Leonard FC, Markey BK. Meticillin-resistant Staphylococcus aureus in animals: a review. Vet J. 2008;175(1):27-36.

18. Hanberger H, Diekema D, Fluit A, Jones R, Struelens M, Spencer R, et al. Surveillance of antibiotic resistance in European ICUs. J Hosp Infect. 2001:48(3):161-76

19. Agvald-Ohman C, Lund B, Edlund C. Multiresistant coagulase-negative staphylococci disseminate frequently between intubated patients in a multidisciplinary intensive care unit. Crit Care. 2004;8(1):R42-7.

20. Mohan U, Jindal N, Aggarwal P. Species distribution and antibiotic sensitivity pattern of coagulase negative staphylococci isolated from various clinical specimens. Indian J Med Microbiol. 2002;20(1):45-6.

21. Akinkunmi E, Lamikanra A. Species Distribution and Antibiotic resistance in coagulase-negative staphylococci colonizing the gastrointestinal tract of children in Ile-Ife, Nigeria. Trop J Pharm Res. 2010;9(1):35-43.

22. Fritsche TR, Sader HS, Jones RN. Comparative activity and spectrum of broad-spectrum $\beta$-lactams (cefepime, ceftazidime, ceftriaxone, piperacillin/ tazobactam) tested against 12,295 staphylococci and streptococci: report from the SENTRY antimicrobial surveillance program (North America: 2001 2002). Diagn Microbiol Infect Dis. 2003;47(2):435-40.

23. Ferreira RBR, Nunes APF, Kokis VM, Krepsky N, de Fonseca LS, de Bastos Mdo CF, et al. Simultaneous detection of the mecA and iles-2 genes in coagulase-negative staphylococci isolated from Brazilian hospitals by multiplex PCR. Diagn Microbiol Infect Dis. 2002;42(3):205-12.

24. Idriss SHE, Foltys V, Tančin V, Kirchnerová K, Tančinová D, Zaujec K. Mastitis pathogens and their resistance against antimicrobial agents in dairy cows in Nitra, Slovakia. Slovak J Anim Sci. 2014:47(1):33-8.

25. Antoniadou A, Kanellakopoulou K, Kanellopoulou M, Polemis M, Koratzanis G, Papademetriou E, et al. Impact of a hospital-wide antibiotic restriction policy program on the resistance rates of nosocomial gram-negative bacteria. Infect Dis (Auckl). 2013:45(6):438-45.

26. Cantas L, Shah SQA, Cavaco LM, Manaia CM, Walsh F, Popowska M, et al. A brief multi-disciplinary review on antimicrobial resistance in medicine and its linkage to the global environmental microbiota. Front Microbiol. 2013;4(10):96.

27. Stefani S, Varaldo PE. Epidemiology of methicillin-resistant staphylococci in Europe. Clin Microbiol Infect. 2003;9(12):1179-86.

28. Tulinski P, Fluit AC, Wagenaar JA, Mevius D, van de Vijver L, Duim B. Methicillin-resistant coagulase-negative staphylococci on pig farms as a reservoir of heterogeneous staphylococcal cassette chromosome mec elements. Appl Environ Microbiol. 2012;78(2):299-304.

29. Hussain Z, Stoakes L, Massey V, Diagre D, Fitzgerald V, El Sayed S, et al. Correlation of oxacillin MIC with mecA gene carriage in coagulasenegative staphylococci. J Clin Microbiol. 2000;38(2):752-4.

30. Hososaka Y, Hanaki H, Endo H, Suzuki Y, Nagasawa Z, Otsuka Y, et al. Characterization of oxacillin-susceptible mecA-positive Staphylococcus aureus: a new type of MRSA. J Infect Chemother. 2007;13(2):79-86.

31. Saeed K, Dryden M, Parnaby R. Oxacillin-susceptible MRSA, the emerging MRSA clone in the UK? J Hosp Infect. 2010;76(3):267-8.

32. Monecke S, Coombs G, Shore AC, Coleman DC, Akpaka P, Borg M, et al. A field guide to pandemic, epidemic and sporadic clones of methicillin-resistant Staphylococcus aureus. PLoS One. 2011;6(4):e17936.

33. Oliveira DC, Tomasz ALH. Secrets of success of a human pathogen: molecular evolution of pandemic clones of meticillin-resistant Staphylococcus aureus. Lancet Infect Dis. 2002;2(3):180-9.

34. Zhang K, McClure J-A, Elsayed S, Conly JM. Novel staphylococcal cassette chromosome mec type, tentatively designated type VIII, harboring class a mec and type 4 ccr gene complexes in a Canadian epidemic strain of methicillin-resistant Staphylococcus aureus. Antimicrob Agents Chemother. 2009;53(2):531-40.

35. Bouchami O, Ben Hassen A, de Lencastre H, Miragaia M. Molecular epidemiology of methicillin-resistant Staphylococcus hominis (MRSHo): low Clonality and reservoirs of SCCmec structural elements. Chaturvedi V, editor PLoS One. 2011:6(7):e21940.

36. Harrison EM, Paterson GK, Holden MTG, Morgan FJE, Larsen AR, Petersen A, et al. A Staphylococcus xylosus isolate with a new mecC allotype. Antimicrob Agents Chemother. 2013;57(3):1524-8. 
37. Katayama Y, Takeuchi F, Ito T, Ma XX, Ui-Mizutani Y, Kobayashi I, et al. Identification in methicillin-susceptible Staphylococcus hominis of an active primordial mobile genetic element for the staphylococcal cassette

chromosome mec of methicillin-resistant Staphylococcus aureus. J Bacteriol. 2003;185(9):2711-22.

38. Herwaldt LA, Geiss M, Kao C, Pfaller MA. The positive predictive value of isolating coagulase-negative staphylococci from blood cultures. Clin Infect Dis. 1996;22(1):14-20.

39. Wang XM, Noble L, Kreiswirth BN, Eisner W, McClements W, Jansen KU, et al. Evaluation of a multilocus sequence typing system for Staphylococcus epidermidis. J Med Microbiol. 2003:52(11):989-98.

40. Miragaia M, Thomas JC, Couto I, Enright MC, de Lencastre H. Inferring a population structure for Staphylococcus epidermidis from multilocus sequence typing data. J Bacteriol. 2007;189(6):2540-52.

Ready to submit your research? Choose BMC and benefit from:

- fast, convenient online submission

- thorough peer review by experienced researchers in your field

- rapid publication on acceptance

- support for research data, including large and complex data types

- gold Open Access which fosters wider collaboration and increased citations

- maximum visibility for your research: over $100 \mathrm{M}$ website views per year 